\title{
Pauli Spin Susceptibility of a Strongly Correlated Two-Dimensional Electron Liquid
}

\author{
A. A. Shashkin, ${ }^{1, *}$ S. Anissimova, ${ }^{1}$ M. R. Sakr, ${ }^{1, \dagger}$ S. V. Kravchenko, ${ }^{1}$ V. T. Dolgopolov, ${ }^{2}$ and T. M. Klapwijk ${ }^{3}$ \\ ${ }^{1}$ Physics Department, Northeastern University, Boston, Massachusetts 02115, USA \\ ${ }^{2}$ Institute of Solid State Physics, Chernogolovka, Moscow District 142432, Russia \\ ${ }^{3}$ Kavli Institute of Nanoscience, Delft University of Technology, 2628 CJ Delft, The Netherlands
}

(Received 24 April 2005; published 24 January 2006)

\begin{abstract}
Thermodynamic measurements reveal that the Pauli spin susceptibility of strongly correlated twodimensional electrons in silicon grows critically at low electron densities - behavior that is characteristic of the existence of a phase transition.
\end{abstract}

DOI: $10.1103 /$ PhysRevLett.96.036403

PACS numbers: 71.30.+h, 73.40.Qv

Presently, theoretical description of interacting electron systems is restricted to two limiting cases: (i) weak electron-electron interactions (small ratio of the Coulomb and Fermi energies $r_{s}=E_{C} / E_{F} \ll 1$, high electron densities) and (ii) very strong electron-electron interactions $\left(r_{s} \gg 1\right.$, very low electron densities). In the first case, conventional Fermi-liquid behavior [1] is established, while in the second case, formation of the Wigner crystal is expected [2] [for recent developments, see Ref. [3] ]. Numerous experiments performed in both three- (3D) and two-dimensional (2D) electron systems at intermediate interaction strengths $\left(1 \lesssim r_{s} \lesssim 5\right)$ have not demonstrated any significant change in properties compared to the weakly interacting regime [see, e.g., Refs. [4,5] ]. It was not until recently that qualitative deviations from the weakly interacting Fermi-liquid behavior (in particular, the drastic increase of the effective electron mass with decreasing electron density) have been found in strongly correlated 2D electron systems $\left(r_{s} \gtrsim 10\right)$ [6]. However, these findings have been based solely on the studies of a kinetic parameter (conductivity), which, in general, is not a characteristic of a state of matter.

The 2D electron system in silicon turns out to be a very convenient object for studies of the strongly correlated regime due to the large interaction strengths $\left(r_{s}>10 \mathrm{can}\right.$ be easily reached) and high homogeneity of the samples estimated (from the width of the magnetocapacitance minima in perpendicular magnetic fields) at about $4 \times$ $10^{9} \mathrm{~cm}^{-2}$ [7]. In this Letter, we report measurements of the thermodynamic magnetization and density of states in such a low-disordered, strongly correlated 2D electron system in silicon. We concentrate on the metallic regime where conductivity $\sigma \gg e^{2} / h$. We have found that in this system, the spin susceptibility of band electrons (Pauli spin susceptibility) becomes enhanced by almost an order of magnitude at low electron densities, growing critically near a certain critical density $n_{\chi} \approx 8 \times 10^{10} \mathrm{~cm}^{-2}$ : behavior that is characteristic in the close vicinity of a phase transition. The density $n_{\chi}$ is coincident within the experimental uncertainty with the critical density $n_{c}$ for the zero-field metal-insulator transition (MIT) in our samples. The nature of the low-density phase $\left(n_{s}<n_{\chi}\right)$ still remains unclear because even in the cleanest of currently available samples, it is masked by the residual disorder in the electron system.

Measurements were made in an Oxford dilution refrigerator on low-disordered (100)-silicon samples with peak electron mobilities of $3 \mathrm{~m}^{2} / \mathrm{Vs}$ at $0.1 \mathrm{~K}$ and oxide thickness $149 \mathrm{~nm}$. These samples are remarkable by the absence of a band tail of localized electrons down to electron densities $n_{s} \approx 1 \times 10^{11} \mathrm{~cm}^{-2}$, as inferred from the coincidence of the full spin polarization field obtained from parallel-field magnetotransport and from the analysis of Shubnikov-de Haas oscillations [the former is influenced by possible band tail of localized electrons, while the latter is not; for more details, see Refs. [6,8,9] ]. This allows one to study properties of a clean $2 \mathrm{D}$ electron system without admixture of local moments [8-10]. The second advantage of these samples is a very low contact resistance (in "conventional" silicon samples, high contact resistance becomes the main experimental obstacle in the lowdensity-low-temperature limit). To minimize contact resistance, thin gaps in the gate metallization have been introduced, which allows for maintaining high electron density near the contacts regardless of its value in the main part of the sample.

For measurements of the magnetization, the parallel magnetic field $B$ was modulated with a small ac field $B_{\text {mod }}$ in the range $0.01-0.03 \mathrm{~T}$ at a frequency $f=$ $0.45 \mathrm{~Hz}$, and the current between the gate and the twodimensional electron system was measured with high precision $\left(\sim 10^{-16}\right.$ A) using a current-voltage converter and a lock-in amplifier. The imaginary (out-of-phase) current component is equal to $i=\left(2 \pi f C B_{\text {mod }} / e\right) d \mu / d B$, where $C$ is the capacitance of the sample and $\mu$ is the chemical potential. By applying the Maxwell relation $d M / d n_{s}=$ $-d \mu / d B$, one can obtain the magnetization $M$ from the measured $i$. A similar technique has been applied by Prus et al. [11] to a 2D electron system in silicon with high level of disorder, in which case the physics of local moments has been mainly studied. As discussed below, the data analysis and interpretation is not quite correct in Ref. [11]; in particular, Prus et al. do not distinguish between the Pauli spin 
susceptibility of band electrons and the Curie spin susceptibility of local moments.

For measurements of the thermodynamic density of states, a similar circuit was used with a distinction that the gate voltage was modulated and thus the imaginary current component was proportional to the capacitance. Thermodynamic density of states $d n_{s} / d \mu$ is related to magnetocapacitance via $1 / C=1 / C_{0}+1 / A e^{2}\left(d n_{s} / d \mu\right)$, where $C_{0}$ is the geometric capacitance and $A$ is the sample area.

A typical experimental trace of $i\left(n_{s}\right)$ in a parallel magnetic field of $5 \mathrm{~T}$ is displayed in Fig. 1. The inset shows magnetization $M\left(n_{s}\right)$ in the metallic phase obtained by integrating $d M / d n_{s}=-d \mu / d B$ with the integration constant $M(\infty)=B \chi_{0}$, where $\chi_{0}$ is the Pauli spin susceptibility of noninteracting electrons. A nearly antisymmetric jump of $i\left(n_{s}\right)$ about zero on the $y$ axis (marked by the black arrow) separates the high- and low-density regions in which the signal is positive and negative $\left[M\left(n_{s}\right)\right.$ is decreasing and increasing], respectively. Such a behavior is expected based on simple considerations. At low densities, all electrons are spin polarized in a magnetic field, so for the simple case of noninteracting $2 \mathrm{D}$ electrons one expects $d \mu / d B=-\mu_{B}$ (at $n_{s} \rightarrow 0$, deep in the insulating regime, the capacitance of the system vanishes and, therefore, the measured current approaches zero). At higher densities, when the electrons start to fill the upper spin subband, $M\left(n_{s}\right)$ starts to decrease, and $d \mu / d B$ is determined by the renormalized Pauli spin susceptibility $\chi$ and is expected to decrease with $n_{s}$ due to reduction in the strength of electron-electron interactions. Finally, in the highdensity limit, the spin susceptibility approaches its "noninteracting" value $\chi_{0}$, and $d \mu / d B$ should approach zero. The onset of complete spin polarization - the electron density $n_{p}$ at which the electrons start to fill the upper

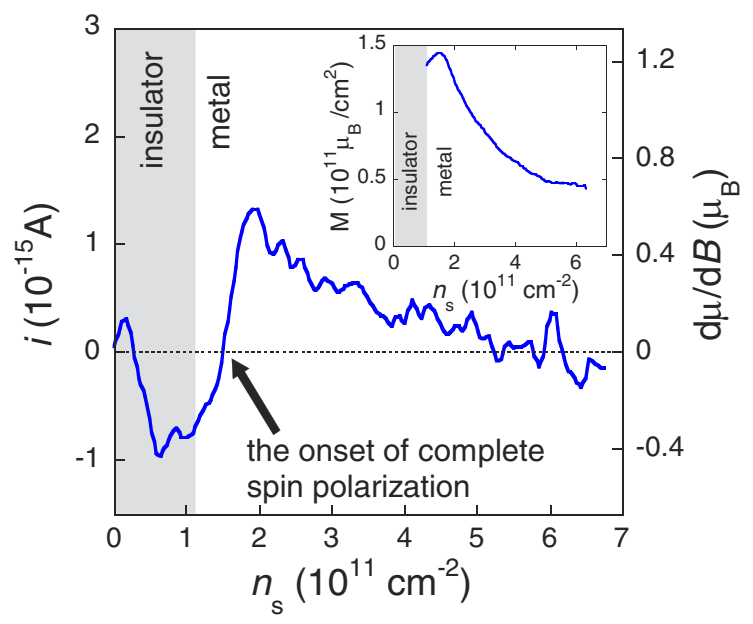

FIG. 1 (color online). Imaginary current component in the magnetization experiment as a function of the electron density in a magnetic field of $5 \mathrm{~T}$ and $T=0.4 \mathrm{~K}$. Grey area depicts the insulating phase. Magnetization vs $n_{s}$ is displayed in the inset. Note that the maximum $M$ is coincident within the experimental uncertainty with $\mu_{B} n_{s}$. spin subband-is given by the condition $d \mu / d B=0$ $\left[M\left(n_{s}\right)\right.$ reaches a maximum], as indicated by the black arrow in the figure. It is important that over the range of magnetic fields used in the experiment $(1.5-7 \mathrm{~T})$, the maximum $M$ coincides within the experimental uncertainty with $\mu_{B} n_{s}$ thus confirming that all the electrons are indeed spin polarized below $n_{p}$. Note, however, that the absolute value of $d \mu / d B$ at $n_{s} \lesssim n_{c}$ is reduced in the experiment. We attribute this to smearing of the minimum in $i\left(n_{s}\right)$ caused by possible influence of the residual disorder in the electron system, which is crucial in and just above the insulating phase, in contrast to the clean metallic regime we focus on here. Another reason for the reduction in $d \mu / d B$ is the electron-electron interactions (due to, e.g., the enhanced effective mass).

In Fig. 2(a), we show a set of curves for the experimental $d \mu / d B$ versus electron density in different magnetic fields. Experimental results in the range of magnetic fields studied do not depend, within the experimental noise, on temperature below $0.6 \mathrm{~K}$ (down to $0.15 \mathrm{~K}$ which was the lowest temperature achieved in this experiment). The onset of full spin polarization shifts to higher electron densities with increasing magnetic field. Grey area depicts the insulating phase, which expands somewhat with $B$ [for more on this, see Ref. [12] ]. Note that the range of magnetic fields used in our experiment is restricted from below by the condition that $d \mu / d B$ crosses zero in the metallic regime. In Fig. 2(b), we show how these curves, normalized by magnetic field, collapse in the partially polarized regime onto a single "master curve." The existence of such scaling verifies proportionality of the magnetization to $B$, confirming that we deal with Pauli spin susceptibility of band electrons, and establishes a common zero level for the experi-
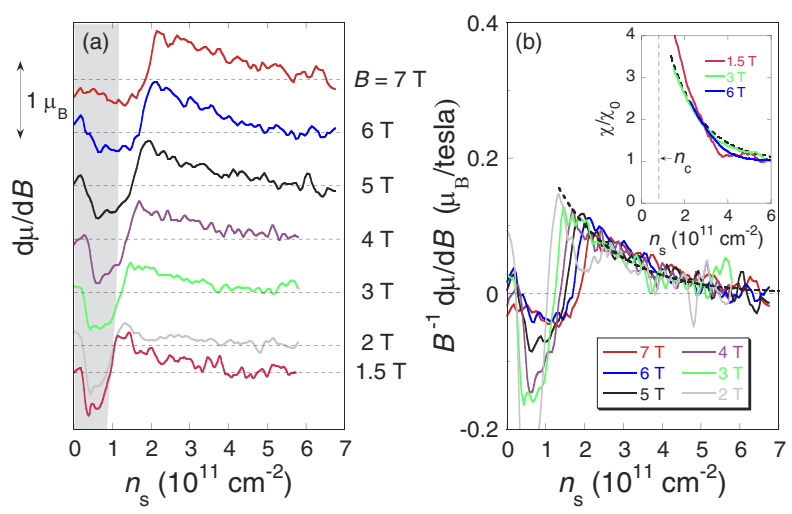

FIG. 2 (color online). (a) The experimental $d \mu / d B$ as a function of electron density in different magnetic fields and $T=$ $0.4 \mathrm{~K}$. The curves are vertically shifted for clarity. Grey area depicts the insulating phase. Note that the onset of full spin polarization in our experiment always takes place in the metallic regime. (b) Scaling of the $d \mu / d B$ curves, normalized by magnetic field magnitude, at high electron densities. The dashed line represents the "master curve." Spin susceptibility obtained by integrating the master curve (dashed line) and raw data at $B=$ $1.5,3$, and $6 \mathrm{~T}$ is displayed in the inset. 
mental traces. Integration of the master curve over $n_{s}$ yields the spin susceptibility $\chi=M / B$, as shown in the inset to Fig. 2(b). Also shown is the spin susceptibility obtained by integration of raw curves at $B=1.5,3$, and $6 \mathrm{~T}$, which, within the experimental error, yield the same dependence.

This method of measuring the spin susceptibility, being the most direct, suffers, however, from possible influence of the unknown diamagnetic contribution to the measured $d \mu / d B$, which arises from the finite width of the 2D electron layer [13]. To verify that this influence is negligible in our samples, we employ another two independent methods to determine $\chi$. The second method is based on marking the electron density $n_{p}$ at which $d \mu / d B=0$ and which corresponds to the onset of complete spin polarization, as mentioned above. The so-determined polarization density $n_{p}(B)$ can be easily converted into $\chi\left(n_{s}\right)$ via $\chi=$ $\mu_{B} n_{p} / B$. Note that in contrast to the value of $d \mu / d B$, the polarization density $n_{p}$ is practically not affected by possible influence of the diamagnetic shift.

The third method for measuring $n_{p}$ and $\chi$, insensitive to the diamagnetic shift, relies on analyzing the magnetocapacitance, $C$. Experimental traces $C\left(n_{s}\right)$ are shown in Fig. 3(a) for different magnetic fields. As the magnetic field is increased, a steplike feature emerges on the $C\left(n_{s}\right)$ curves and shifts to higher electron densities. This feature corresponds to the thermodynamic density of states abruptly changing when the electrons' spins become completely polarized. To see the steplike feature more clearly, in Fig. 3(b) we subtract the $C\left(n_{s}\right)$ curves for different magnetic fields from the reference $B=0$ curve. The fact that the jumps in $C$ (as well as in $d \mu / d B$ ) are washed out much stronger than it can be expected from possible inhomogeneities in the electron density distribution [about $4 \times 10^{9} \mathrm{~cm}^{-2}$ [7]] points to the importance of electronelectron interactions. Since the effects of interactions are different in the fully and partially polarized regimes, it is natural to mark the onset of full spin polarization at the beginning of the interaction-broadened jump, as indicated
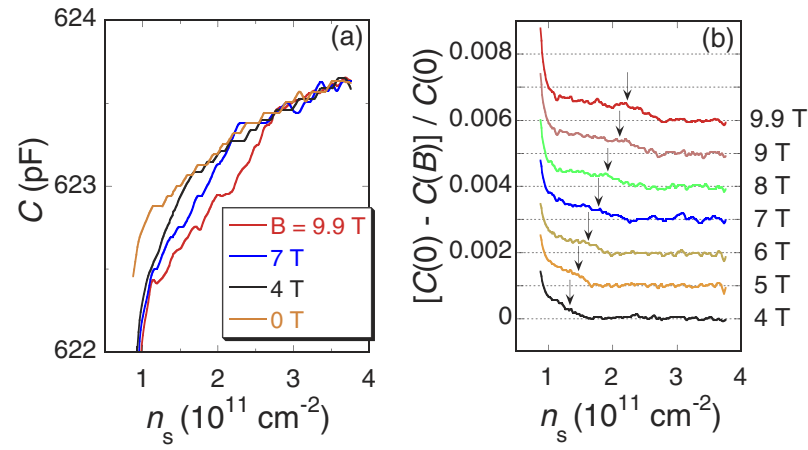

FIG. 3 (color online). (a) Magnetocapacitance vs electron density for different magnetic fields. (b) Deviation of the $C\left(n_{s}\right)$ dependences for different magnetic fields from the $B=0$ reference curve. The traces are vertically shifted for clarity. The onset of full spin polarization is indicated by arrows. by arrows in the figure. In case the residual disorder does contribute to the jump broadening, we extend error bars to the middle of the jump, which yields an upper boundary for the onset of full spin polarization.

In Fig. 4, we show the summary of the results for the Pauli spin susceptibility as a function of $n_{s}$, obtained using all three methods described above. The excellent agreement between the results obtained by all of the methods establishes that a possible influence of the diamagnetic shift is negligible [14] and, therefore, the validity of the data including those at the lowest electron densities is justified. There is also good agreement between these results and the data obtained by the transport experiments of Ref. [7]. This adds credibility to the transport data and confirms that full spin polarization occurs at the field $B_{c}$; however, we note again that evidence for the phase transition can only be obtained from thermodynamic measurements. The magnetization data extend to lower densities than the transport data, and larger values of $\chi$ are reached, exceeding the "noninteracting" value $\chi_{0}$ by almost an order of magnitude. The Pauli spin susceptibility behaves critically close to the critical density $n_{c}$ for the $B=0$ metal-insulator transition [15]: $\chi \propto n_{s} /\left(n_{s}-n_{\chi}\right)$. This is in favor of the occurrence of a spontaneous spin polarization [either Wigner crystal [16] or ferromagnetic liquid] at low $n_{s}$, although in currently available samples, the formation of the band tail of localized electrons at $n_{s} \lesssim n_{c}$

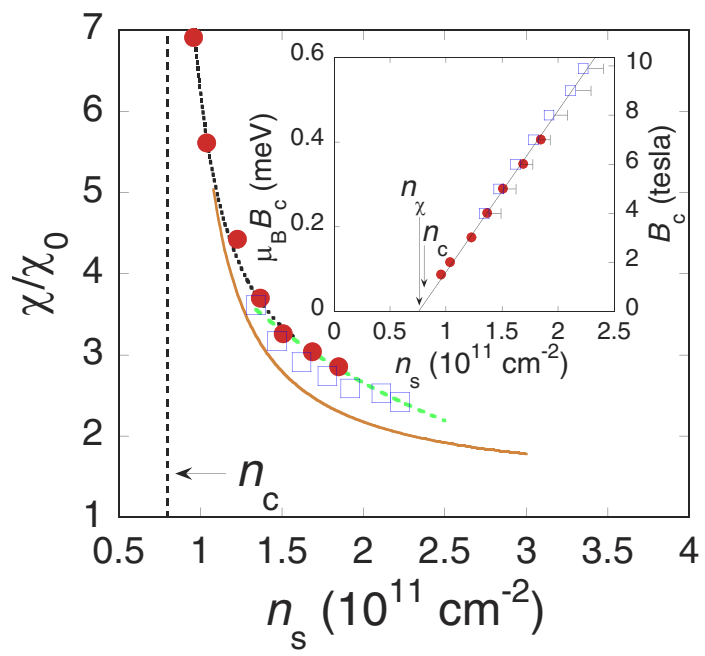

FIG. 4 (color online). Dependence of the Pauli spin susceptibility on electron density obtained by all three methods described in text: integral of the master curve (dashed line), $d \mu / d B=0$ (circles), and magnetocapacitance (squares). The dotted line is a guide to the eye. Also shown by a solid line is the transport data of Ref. [7]. Inset: polarization field as a function of the electron density determined from the magnetization (circles) and magnetocapacitance (squares) data. The symbol size for the magnetization data reflects the experimental uncertainty, and the error bars for the magnetocapacitance data extend to the middle of the jump in $C$. The data for $B_{c}$ are described by a linear fit which extrapolates to a density $n_{\chi}$ close to the critical density $n_{c}$ for the $B=0$ MIT. 
conceals the origin of the low-density phase. In other words, so far, one can only reach an incipient transition to a new phase.

The dependence $B_{c}\left(n_{s}\right)$, determined from the magnetization and magnetocapacitance data, is represented in the inset to Fig. 4. The two data sets coincide and are described well by a common linear fit which extrapolates to a density $n_{\chi}$ close to $n_{c}$. We emphasize that in the low-field limit $(B<1.5 \mathrm{~T})$, the jump in $d \mu / d B$ shifts to the insulating regime, which does not allow us to approach closer vicinity of $n_{\chi}$ : based on the data obtained in the regime of strong localization, one would not be able to make conclusions concerning properties of a clean metallic electron system which we are interested in here. Clearly, the fact that the linear $B_{c}\left(n_{s}\right)$ dependence persists down to the lowest electron densities achieved in the experiment confirms that we always deal with the clean metallic regime.

Finally, we would like to clarify the principal difference between our results and those of Ref. [11]. In the sample used by Prus et al., the critical density $n_{c}$ for the $B=0$ MIT was considerably higher than in our samples caused by high level of disorder, and the band tail of localized electrons was present at all electron densities [11]. As a result, the crucial region of low electron densities, in which the critical behavior of the Pauli spin susceptibility occurs, falls within the insulating regime where the physics of local moments dominates [8-10]. Indeed, Prus et al. have found sublinear $M(B)$ dependence characteristic of local moments, and the extracted spin susceptibility in their sample has a Curie temperature dependence [9]. This is the case even at high electron densities, where metallic behavior might be expected instead. Such effects are absent in our samples: the spin susceptibility (in the partially polarized system) is independent of the magnetic field and temperature, confirming that we deal with Pauli spin susceptibility of band electrons.

In summary, the Pauli spin susceptibility has been determined by measurements of the thermodynamic magnetization and density of states in a low-disordered, strongly correlated 2D electron system in silicon. It is found to behave critically near the zero-field MIT, which is characteristic of the existence of a phase transition.

We gratefully acknowledge discussions with S. Chakravarty, D. Heiman, N. E. Israeloff, R. S. Markiewicz, and M. P. Sarachik. One of us (S. V. K.) would like to thank B. I. Halperin for suggesting this method to measure spin susceptibility. We would also like to thank A. Gaidarzhy and J. B. Miller for technical assistance and C. M. Marcus and P. Mohanty for an opportunity to use their microfabrication facilities. This work was supported by NSF Grant No. DMR-0403026, PRF Grant No. 41867-AC10, the RFBR, RAS, and the Programme "The State Support of Leading Scientific Schools."
*Permanent address: Institute of Solid State Physics, Chernogolovka, Moscow District 142432, Russia.

${ }^{\dagger}$ Present address: Department of Physics and Astronomy, UCLA, Los Angeles, CA 90095, USA.

[1] L. D. Landau, Sov. Phys. JETP 3, 920 (1957).

[2] E. Wigner, Phys. Rev. 46, 1002 (1934).

[3] B. Tanatar and D. M. Ceperley, Phys. Rev. B 39, 5005 (1989); G. Benenti, X. Waintal, and J.-L. Pichard, Phys. Rev. Lett. 83, 1826 (1999); C. Attaccalite, S. Moroni, P. Gori-Giorgi, and G. B. Bachelet, Phys. Rev. Lett. 88, 256601 (2002).

[4] H. von Löhneysen, Adv. Solid State Phys. 30, 95 (1990).

[5] T. Okamoto, K. Hosoya, S. Kawaji, and A. Yagi, Phys. Rev. Lett. 82, 3875 (1999); J. Zhu, H. L. Stormer, L. N. Pfeiffer, K. W. Baldwin, and K. W. West, Phys. Rev. Lett. 90, 056805 (2003).

[6] S. V. Kravchenko and M. P. Sarachik, Rep. Prog. Phys. 67, 1 (2004); A. A. Shashkin, Phys. Usp. 48, 129 (2005).

[7] A. A. Shashkin, S. V. Kravchenko, V. T. Dolgopolov, and T. M. Klapwijk, Phys. Rev. Lett. 87, 086801 (2001).

[8] V. T. Dolgopolov and A. Gold, Phys. Rev. Lett. 89, 129701 (2002).

[9] A. Gold and V. T. Dolgopolov, J. Phys. Condens. Matter 14, 7091 (2002).

[10] N.F. Mott and E. A. Davis, Electronic Processes in NonCrystalline Materials (Clarendon, Oxford, 1971).

[11] O. Prus, Y. Yaish, M. Reznikov, U. Sivan, and V. Pudalov, Phys. Rev. B 67, 205407 (2003).

[12] A. A. Shashkin, S. V. Kravchenko, and T. M. Klapwijk, Phys. Rev. Lett. 87, 266402 (2001).

[13] To deal with this problem, the "subtraction of the diamagnetic contribution" was suggested in Ref. [11]. The diamagnetic contribution $\Delta$ was determined in the partially polarized regime as the difference between the direct magnetization data ("mag") and the data obtained from Shubnikov-de Haas oscillations ("SdH"): $\Delta=$ mag$\mathrm{SdH}$. The experimental data were then corrected by $\Delta$. We find this procedure meaningless as it essentially results in replacing the magnetization data by Shubnikov-de Haas data: $\operatorname{mag}-\Delta=\operatorname{mag}-(\operatorname{mag}-\mathrm{SdH})=\mathrm{SdH}$. In fact, the difference between magnetization and Shubnikov-de Haas data in their experiment is likely to be due to the presence of a band tail of localized electrons at all electron densities in their sample.

[14] Since the diamagnetic shift decreases with increasing $n_{s}$ and/or decreasing $B$, it may in principle be noticeable at the lowest $n_{s}$ and highest $B$ used in the experiment. Comparing low- and high-field curves shown in Fig. 2(a), we estimate that at $n_{s} \sim 1 \times 10^{11} \mathrm{~cm}^{-2}$ and $B=7 \mathrm{~T}$, the contribution of the diamagnetic shift is less than $0.2 \mu_{B}$.

[15] The critical density for the MIT was determined from transport measurements [see Refs. [6,12]].

[16] The fact that $B_{c} \rightarrow 0$ as $n_{s} \rightarrow n_{c}$ speaks in favor of the strongly correlated liquid being close to the crystal [17].

[17] B. Castaing and P. Nozières, J. Phys. (Paris) 40, 257 (1979). 